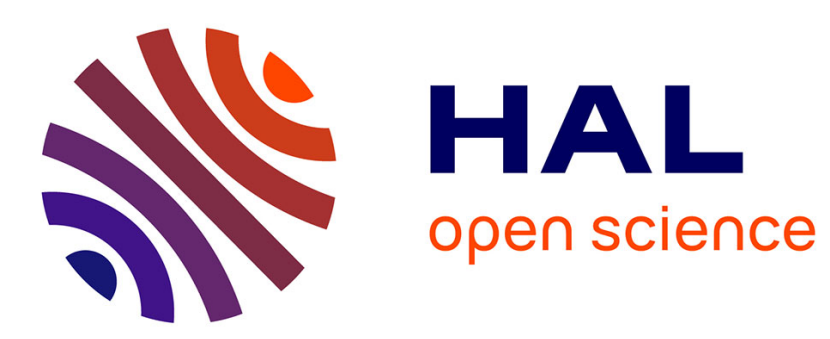

\title{
Sortir de l'impasse épistémologique. Nouveaux médicaments et savoirs traditionnels
}

Laurent Pordié

\section{To cite this version:}

Laurent Pordié. Sortir de l'impasse épistémologique. Nouveaux médicaments et savoirs traditionnels. Sciences Sociales et Santé, 2012, 30 (2), pp.93-103. hal-01045143

\section{HAL Id: hal-01045143 \\ https://hal.science/hal-01045143}

Submitted on 24 Jul 2014

HAL is a multi-disciplinary open access archive for the deposit and dissemination of scientific research documents, whether they are published or not. The documents may come from teaching and research institutions in France or abroad, or from public or private research centers.
L'archive ouverte pluridisciplinaire HAL, est destinée au dépôt et à la diffusion de documents scientifiques de niveau recherche, publiés ou non, émanant des établissements d'enseignement et de recherche français ou étrangers, des laboratoires publics ou privés. 
Sciences Sociales et Santé, Vol. 30, $n^{\circ} 2$, juin 2012

\section{Sortir de l'impasse épistémologique. Nouveaux médicaments et savoirs traditionnels}

\section{Commentaire}

Laurent Pordie**

Non, il ne s'agit pas d'un oxymore. Les médicaments traditionnels peuvent également constituer des nouveautés. Comme nous l'a appris Leslie il y a plus de trois décennies, les médecines dites traditionnelles se modifient au cours de leur histoire, de leur contact avec la modernité, la science et la technologie (Leslie, 1976). Les facteurs sociaux tiennent un rôle fondamental dans la transformation historique et épistémologique des médecines. La remarquable étude conduite par Scheid sur le devenir et les pratiques thérapeutiques de lignées médicales chinoises au cours de quatre siècles montre bien la fluidité de la tradition, d'un praticien ou d'une école à l'autre, ainsi que les modifications et parfois les bouleversements qui renouvellent, au fil du temps, les pratiques et les savoirs relatifs au corps et à la santé (Scheid, 2007). Les trajectoires complexes des savoirs thérapeutiques et de la matière médicinale dans le développement historique des médecines révisent les traditions établies et ouvrent des espaces propices aux innovations (Hsu, 2001). Les diverses modalités de traitements thérapeutiques, dont le médicament est un exemple, ne font

* Laurent Pordié, anthropologue, CNRS (UMR 8211/CERMES3), Campus CNRS, 7,rue Guy-Môquet, 94801 Villejuif Cedex, France; pordie@vjf.cnrs.fr 
évidemment pas dans ce domaine figure d'exception. Leur variabilité est à la fois spatiale et temporelle. Les thérapeutes font preuve d'innovation, intégrant certaines idées ou certaines substances médicinales dans leur arsenal thérapeutique et en rejetant d'autres, créant ainsi des traitements sur mesure.

La globalisation a particulièrement accéléré ces processus. La libéralisation des marchés, l'intensification des échanges transnationaux et les circulations d'humains, d'idées et d'objets infléchissent les processus de construction des médecines (Alter, 2005 ; Bode, 2008 ; Pordié, 2011 a, 2011b). Encore en marge des sociétés à l'échelle planétaire il y a deux décennies, ces nouvelles formes thérapeutiques sont aujourd'hui positionnées au centre des systèmes sanitaires. Elles intègrent toujours des références à la «tradition » - qui peut être géographiquement ou temporellement éloignée, effective ou inventée, très localisée ou plus universelle - tout en contribuant à produire de nouvelles pratiques. La tradition est une source importante de légitimation pour ces nouveaux médicaments traditionnels (et pour ceux qui les produisent et en font commerce), lesquels convoquent par ailleurs la science moderne, la technologie, le bien-fondé écologique, ou bien les bénéfices sociaux, économiques ou politiques (dans le cadre de programmes de "développement sanitaire »). Les registres de légitimité sont ainsi diversifiés.

Cette situation caractérise ce qu'E. Simon et M. Egrot ont choisi de dénommer les médicaments néotraditionnels dans leur étude au Bénin. Les " médicaments traditionnels améliorés » (MTA) forment l'exemple immédiatement cité par ces auteurs. Largement promus dans les programmes de revalorisation des traditions de soins, tant par les organisations bi- ou multi-latérales que par les ONG et les sociétés savantes, en ethnopharmacologie notamment, ces nouveaux médicaments "s'inspirent de la culture matérielle du médicament pharmaceutique ; ils ont su trouver un contexte favorable à leur développement grâce à la marchandisation des produits de santé mais aussi du fait de l'accélération et de l'extension de leur circulation dans le contexte de la mondialisation ». Ces médicaments font l'objet de recherches cliniques portant sur leur toxicité ou leur efficacité biologique et sont majoritairement indiqués pour des pathologies définies dans le langage biomédical. Ces avatars des traditions béninoises sont des marchandises hybrides, situées à la frontière du remède et du médicament. Ce type de produit est tout à fait cosmopolite. Les médicaments néotraditionnels se retrouvent sous des formes diverses au Tibet, en Suisse ou encore en Inde (Banerjee, 2004 ; Pordié, 2008).

Mais en utilisant le qualificatif " néotraditionnel », E. Simon et M. Egrot entendent aussi autre chose : ils incluent de façon convaincante 
la «phytothérapie importée » et les « compléments alimentaires » (1). Bien que la généalogie de ces produits ne se fonde pas sur des traditions locales, cette assimilation conceptuelle est rendue possible par l'approche des auteurs qui ont choisi de s'intéresser à la façon dont leurs interlocuteurs perçoivent, organisent et classifient les produits thérapeutiques. L'originalité de leur article est de montrer les logiques du regroupement de produits autrement considérés comme appartenant à des catégories distinctes - pour les besoins de la démonstration, la question de leurs spécificités et de la variabilité des représentations locales à leur égard est cependant laissée en suspens, alors qu'elle aurait pu soulever quelques questions importantes. Les auteurs étudient le positionnement de ces produits dans l'espace des officines, la mobilisation de diverses traditions régionales ou étrangères (comme dans le cas de la phytothérapie chinoise) et leurs réseaux de diffusion et de circulation. Dans chacun de ces cas, les MTA, la phytothérapie importée et les compléments alimentaires suivent des itinéraires souvent identiques et sont distingués des "médicaments pharmaceutiques » (biomédicaments). Plus encore, cette proximité conceptuelle et physique bénéficie à l'ensemble de ces produits. Une plante importée dont les mérites sont reconnus en phytothérapie étrangère encourage, par exemple, la consommation d'espèces endémiques apparentées, qui pourront ainsi entrer dans la composition de MTA ou de "phytothérapie locale ». À l'inverse, le recours à la tradition pour ces groupes de produits, même lorsqu'il s'agit de recours rhétorique, est un langage partagé qui facilite les fertilisations croisées. Les frontières se confondent et s'estompent.

Les auteurs concluent sur les mérites de la catégorie "médicaments néotraditionnels », en termes opératoires et théoriques, bien qu'elle constitue plutôt une catégorie descriptive. Il est inutile de s'arrêter plus longuement sur ce point. Ce qui va animer le cour de ce commentaire relève du biais social et culturel qui fait à la fois la force et la faiblesse de l'argument. "L'adjectif "néotraditionnel", nous dit-on, ne qualifie pas la culture matérielle de l'objet mais renvoie (...) à l'environnement social et culturel dans lequel il fonctionne. "Pourtant, tant de choses se passent, se disent et se lisent dans la matière, dans les substances médicinales, dans les formulations médicamenteuses. À mon sens, la « culture matérielle » de l'objet est une caractéristique centrale des médicaments néotraditionnels car elle renvoie au domaine du savoir thérapeutique et à ses

(1) Les auteurs proposent aussi d'inclure les « médicaments pharmaceutiques aux usages non conventionnels », comme l'indique la dernière phrase du texte, mais sans pour autant fournir des éléments d'analyse qui permettraient de comprendre clairement cette inclusion. 
modulations. C'est précisément dans l'objet qui vise à guérir que la valeur thérapeutique et ses significations prennent corps et se transforment. C'est dans la matière que le médicament néotraditionnel est façonné, tout autant que dans le monde social et culturel. Car la matière n'est pas pleine et inviolable. L'objet est aussi un réceptacle. La matérialité contient paradoxalement des espaces, qui permettent à l'objet-médicament d'exister comme tel dans divers environnements épistémologiques en les absorbant tout ou partie. Chacun de ces environnements produit diverses formes de connaissances thérapeutiques, qui produisent les objets destinés à soigner et leur attribuent du sens, de la valeur et du pouvoir dans un contexte et dans une période donnés. La citation rapportée plus haut occulte un aspect fondamental de ces nouveaux médicaments : leur dimension épistémologique.

Proposons donc une grille de lecture basée sur le savoir afin de compléter l'analyse d'E. Simon et M. Egrot. Je retiens dans cet objectif trois pistes de réflexion : la mobilisation des savoirs, les glissements de catégories et les alternatives au modèle moléculaire de l'innovation pharmaceutique. Comme l'indiquent les auteurs de l'article, les médicaments traditionnels sont supposés être " améliorés » par diverses méthodes et techniques qui relèvent essentiellement des sciences pharmaceutiques et biomédicales : galénique moderne, tests de contrôle de qualité, toxicologie, essais cliniques, etc. Signalons que ce type de médicaments se retrouve évidemment en Inde, mon terrain d'enquête, mais ni la formule " médicaments traditionnels améliorés " ni un équivalent dans une des langues nationales n'existe à grande échelle dans le pays. Les locuteurs qui s'en rapprochent choisiront plutôt de parler de pratiques traditionnelles "modernisées ». Cependant, l'épineuse question de la hiérarchie des savoirs en contexte postcolonial n'est évacuée dans aucun des cas. Reconnaître la science moderne comme étant le moyen le plus efficace d'améliorer la tradition demeure problématique car il subordonne les savoirs qui la fondent (l'utilisation de la science pour valider une tradition thérapeutique dans un langage médical plus universel est une démarche perçue comme étant très différente). À bien des égards, le médicament néotraditionnel rend compte des différences de statut entre diverses formes de savoir et cet aspect pourrait également le définir. Il en va de même pour les passages multiples d'un savoir thérapeutique à l'autre qui caractérisent les MTA : compositions de plantes africaines indiquées pour l'hépatite B ou l'arthrose, pour "remonter l'immunité » ou accélérer quelque activité enzymatique. Le médicament néotraditionnel ainsi conçu serait donc aussi caractérisé par une hiérarchisation des savoirs qui lui donnent naissance et un ensemble de traductions, parfois hasardeuses, entre la biomédecine et d'autres univers thérapeutiques. 
Cette définition partielle, me reprochera-t-on, n'inclut pas la «phytothérapie importée » ou les "compléments alimentaires ». Il faut, pour en comprendre pleinement la portée, s'intéresser de plus près à ce que nous disent les auteurs. On apprend que la phytothérapie d'importation provient notamment d'Amérique et de Chine. Une rapide généalogie de la "phytothérapie chinoise » en Afrique révèle le glissement terminologique et épistémologique dont elle souffre. En réalité, si l'usage de préparations à base de plantes médicinales est bien patent en médecine chinoise - aux côtés de l'acupuncture et d'autres pratiques thérapeutiques -, elle se situe dans un cadre théorique et épistémologique défini et très élaboré. Or, tout cela se perd lorsque ces produits se déplacent géographiquement et sont ramenés à une sorte de neutralité phytothérapeutique. Le savoir thérapeutique chinois disparaît pour ne laisser subsister de lui qu'une trace végétale. Les plantes de Chine sont prescrites au Bénin pour des indications biomédicales dans une équation qui associe la plante à un symptôme ou à une maladie définie par la biomédecine et non à un désordre physiologique correspondant à une condition individuelle diagnostiquée et formulée dans les termes propres de la médecine chinoise. Là encore, l'écart entre divers domaines du savoir est abyssal. Les Béninois sont en présence de la partie congrue d'un savoir médical autrement plus complexe.

La démonstration fonctionne également en inversant les données $d u$ problème. La phytothérapie européenne, que le texte indique comme également présente au Bénin, est réappropriée par des entrepreneurs ou des commerciaux locaux. En Europe, cette phytothérapie est essentiellement disponible en pharmacie et fonctionne sur un mode très biomédicalisé. Or, l'arrivée de ces médicaments dans d'autres pays et d'autres cultures laisse aussi émerger des savoirs hybrides, inédits, conciliant les usages de la phytothérapie avec les pratiques et les croyances préexistantes à sa venue. Ce type de médicaments néotraditionnels - ici, la phytothérapie dans un nouveau contexte - n'échappe pas à la confrontation des savoirs, à leur traduction et leur hiérarchisation. Nous sommes en droit de nous demander s'il s'agit toujours bien de phytothérapie européenne ou chinoise.

Une caractéristique importante des médicaments néotraditionnels est, en effet, de changer de statut selon les contextes (Pordié, 2008). C'est ainsi que les médicaments ayurvédiques qui sont en Inde considérés comme issus des " médecines traditionnelles » sont catégorisés en Angleterre comme produits de "médecine alternative » et, aux États-Unis, comme " compléments alimentaires ", selon les contextes règlementaires de ces deux pays. Cette situation permet d'expliquer le fait que, dans de nombreuses contrées, les "compléments alimentaires » sont régulière- 
ment prescrits dans le traitement de diverses pathologies et utilisés comme médicaments. En fait, comme le soulignent E. Simon et M. Egrot, il s'agit souvent de médicaments camouflés, lorsque l'environnement légal du pays d'accueil n'est pas favorable à leur entrée sur le marché. Ce glissement de catégorie - qui ne signifie pas que ces catégories soient fondues entre elles - autorise ou facilite l'importation (2). Il entraîne aussi, comme indiqué plus haut, un ré-ordonnancement des savoirs associés à ces médicaments lorsqu'ils se retrouvent dans des contextes sociaux et culturels renouvelés.

Pour certains auteurs spécialistes de l'Inde, les transformations des savoirs et des pratiques consubstantielles à l'essor contemporain de ces nouveaux médicaments altèrent vivement les Grandes Traditions, dont ils semblent regretter la dilution au profit de pratiques hybrides à forte portée commerciale (Lau, 2000 ; Selby, 2005 ; Zysk, 2001). D'autres collègues sont allés jusqu'à prédire la disparition des traditions indiennes dans les nouveaux médicaments qui en sont pourtant issus. Ainsi, Banerjee note que "les (nouveaux) médicaments ayurvédiques ne sont plus distinguables des autres médicaments. Ils ne portent plus les marques du système de savoir qui les fondent (...). La capacité de l'âyurveda à se positionner comme système "alternatif" s'érode graduellement " (Banerjee, 2008 : 201-202). Ces affirmations ne sont pourtant pas vérifiées lorsque l'on examine le domaine des savoirs, leurs mobilisations et leurs reformulations. Intéressons-nous maintenant aux nouveaux médicaments ayurvédiques et à leur production afin de cerner leurs particularités pharmaceutiques (3) Nous constaterons que ces médicaments constituent bien une forme de "modernité alternative » (Knauft, 2002).

Depuis le début $d u X X^{e}$ siècle, l'industrie ayurvédique est le principal producteur de nouveaux médicaments traditionnels en Inde. Elle alimente aujourd'hui un nouveau marché intérieur de la santé destiné aux classes moyennes supérieures, elle facilite l'export de produits thérapeutiques et pourvoit aux besoins des patients internationaux, dont le nombre est en augmentation constante depuis plus d'une décennie. Cette industrie participe au développement ciblé du secteur sanitaire national (manufactures pharmaceutiques, hôpitaux super spécialisés, industrie du bien-être et voyages médicaux, biotechnologies, recherche clinique) et bénéficie des transformations structurelles, de l'avancée technologique et d'une main-d'œuvre à faible coût. L'évolution parallèle de ces secteurs contribue non seulement à modifier l'offre sanitaire nationale, mais transforme

(2) Voir Janes (2002), dans le cas de médicaments tibétains aux États-Unis.

(3) Les résultats présentés ci-après sont issus de recherches conduites dans le cadre du programme "Pharmasud», financé par l'Agence nationale de la recherche. 
également l'image du pays au niveau mondial, dans un domaine, celui de la santé, longtemps considéré comme médiocre. Les médecines indiennes, âyurveda en particulier, sont au cour du marché mondial des thérapies alternatives et une proportion significative de leur production en médicaments vise l'export. Ces médicaments ayurvédiques de dernière génération inondent le marché indien comme international.

L'extension des procédures de production pharmaceutique et de standardisation des pratiques de laboratoire en Inde n'est cependant pas une réplique du système d'innovation dominant dans les pays du Nord. Cela est d'autant moins le cas que ce secteur des technosciences, fondé sur la synthèse chimique de substances actives et sur l'économie propriétaire du brevet, est lui-même entré en crise en raison des coûts croissants et des rendements décroissants de l'innovation basée sur le screening (Gofraind et Ardaillou, 2007). Dans le cas des substances végétales et des savoirs savants de l'âyurveda, ce type de production de connaissances basé sur l'extraction différentielle et la synthèse de principes actifs est une "démarche intellectuellement réductionniste »(Zimmermann, 2011). Elle ne rend pas davantage compte des régimes d'innovation pharmaceutique qui caractérisent actuellement l'Inde, comme dans le cas des nouveaux médicaments traditionnels. Ces régimes se construisent en dehors du paradigme moléculaire du screening pharmaceutique et des biotechnologies qui domine le milieu de la pharmacie depuis la Seconde Guerre mondiale. On remanie, en Inde, à des fins industrielles, des savoirs issus de pratiques médicales locales mêlant thérapies traditionnelles et biomédecine. La généalogie et les usages de ces nouveaux médicaments traversent respectivement les paradigmes médicaux et les frontières sociopolitiques.

Les processus d'innovation de l'industrie ayurvédique sont caractérisés par un « régime de la reformulation » (Pordié et Gaudillière, 2012). La médecine ayurvédique réinvente ses remèdes et emprunte pour cela à divers ordres de pensées scientifiques et médicales, à diverses techniques qui relèvent de la galénique moderne, de la biomédecine ou des traditions indiennes. Essentiellement basée sur ce que ses acteurs appellent une rétro-ingénierie, ce modèle consiste à reformuler et à simplifier les compositions médicamenteuses ayurvédiques afin de créer de nouveaux médicaments «traditionnels » pour les désordres biomédicaux d'une clientèle cosmopolite. Une séquence typique de rétro-ingénierie est composée de l'identification des symptômes (et des marqueurs biologiques) associés dans la littérature biomédicale à l'indication (ou la maladie) visée, la recherche d'une équivalence dans les entités de la médecine ayurvédique, l'inventaire des formulations mentionnant ces entités, la définition d'une formule consensus simplifiée (comprenant au maximum une demi-douzaine d'ingrédients) et la sélection d'un protocole de production et de 
tests (éventuellement biologiques) de cette formulation. Cette démarche nécessairement approximative (incommensurabilité des paradigmes médicaux, difficultés de traduction linguistique et problème d'équivalence conceptuelle, variabilités des identifications botaniques dans les textes de références, etc.) est une démarche très complexe qui implique des passages multiples d'un registre de savoirs et de significations dans un autre (et donc un enchaînement de traductions), dont chaque étape demande à être scrupuleusement étudiée aux niveaux pratique et épistémologique.

L'innovation par reformulation n'est ni une intégration des préparations de plantes à la biomédecine, ni une simple adaptation de pratiques traditionnelles à un contexte industriel, mais une réinvention du médicament traditionnel qui emprunte, pour se construire, à des paradigmes médicaux parfois très éloignés. Le régime de la reformulation est un travail de recomposition qui s'appuie sur des dispositifs singuliers de prospection des savoirs et d'industrialisation des remèdes. La reformulation des médecines ainsi entendue recentre les savoirs et les pratiques de préparation sur les propriétés d'une matière médicinale complexe produite et vendue à une échelle de masse pour des usages qui mêlent les cultures médicales. Le terme de "reformulation » vise ainsi à insister sur la place qu'occupe la formulation médicamenteuse dans ces changements. Cette reformulation des médecines est à la fois l'objet et le produit de pratiques industrielles visant à produire de nouveaux médicaments à base de substances naturelles en faisant largement appel aux catégories diagnostiques de la biomédecine. La manière dont les savoirs sont réarticulés dans l'invention et la production de ce type de produit caractérise les médicaments néotraditionnels.

Ainsi, la création de ces nouveaux médicaments ne fait pas que "s'inspirer de la culture matérielle du médicament pharmaceutique » de manière simplement mimétique, comme cela semble être le cas au Bénin, elle en offre la critique et en permet le renouvellement. C'est en étudiant la façon dont les savoirs se construisent que surgit la particularité de ces produits. Le régime d'innovation qui caractérise l'industrie ayurvédique est un exemple de pharmaceuticalisation (Banerjee, 2008) qui demeure spécifique à l'âyurveda. Cette spécificité apparaît clairement dès lors que l'on observe les réseaux dans lesquels se créent, se diffusent et circulent ces médicaments. En effet, ils s'insèrent dans le réseau sociotechnique de l'âyurveda contemporain - un réseau qui comprend également des vaidyas (praticiens ayurvédiques), des pharmacologues, des pharmacognostes, des chimistes, des graphistes, des commerciaux, des examinateurs de brevets, des administrateurs de santé publique, etc. Bien que le réseau des médicaments ayurvédiques inclue des spécialistes biomédicaux, son déplacement vers le réseau sociotechnique de la biomédecine ne se pro- 
duit pas. Ces produits hybrides et leur mode de production sont spécifiques à l'âyurveda d'aujourd'hui. Une raison évidente est que les manufactures qui créent ces médicaments sont historiquement ancrées dans l'âyurveda ; elles se posent comme concurrentes à la biomédecine et revendiquent leur résistance. Beaucoup plus significatif cependant est le fait que les formes d'innovations qui caractérisent les nouveaux médicaments ayurvédiques sont exclusivement basées sur des formulations médicamenteuses à base de plantes. Ces innovations se distinguent du paradigme de l'innovation pharmaceutique et du recours aux entités moléculaires, et rendent donc difficile, voire impossible, de "réduire matériellement (ces) médicaments aux constituants du réseau (sociotechnique de la biomédecine) »(Lei, 1999 : 345). Ces nouveaux médicaments constituent une forme d'altérité critique. Ils sont conçus, commercialisés et consommés en tant qu'âyurveda, en dépit de l'effort mené pour moderniser cette médecine. Cette situation présente une différence fondamentale avec les nouveaux médicaments de médecine chinoise étudiés par Hsu (2009), qui, non seulement comprennent des préparations de plantes, mais aussi des molécules isolées, comme dans le cas de l'antipaludique nommé artésunate. Dans ce cas, les "nouveaux médicaments chinois» entrent dans l'espace de la biomédecine et ne fournissent plus le même type de critique et le même type d'alternative.

Que les médicaments néotraditionnels renvoient au social et au culturel pour E. Simon et M. Egrot ou soient la configuration matérielle d'un argument politique - le néotraditionalisme - visant à favoriser la reconnaissance d'une pratique médicale aux niveaux social et médical, nationaux et internationaux (Pordié, 2008), ils n'en sont pas moins des formes de savoirs. Ces formes de savoir se donnent à lire dans la matière ellemême, dans les substances, dans les formulations médicamenteuses. Le cas de l'Inde et, je l'espère, mes commentaires profanes sur l'Afrique de l'Ouest, ont permis de saisir l'importance de cette approche - j'insiste sur le fait qu'elle n'est pas exclusive; ce commentaire est un complément d'analyse. Elle permet toutefois de dégager les voies de sortie d'une double impasse épistémologique. La première impasse relève de l'analyse des médicaments néotraditionnels en termes sociaux, politiques et culturels ; elle dissimule les dimensions relatives à la production et la configuration des connaissances comme caractéristiques de ces médicaments. La seconde impasse épistémologique concerne le paradigme moléculaire de la recherche pharmaceutique et la crise contemporaine de l'innovation qui frappe ce secteur. Le régime d'innovation qui caractérise les nouveaux médicaments ayurvédiques réhabilite la polypharmacie ; il offre une alternative à l'économie de l'invention pharmaceutique. Le régime de la reformulation et son hétérodoxie épistémologique permettent ainsi 
d'envisager de nouvelles dynamiques de la globalisation pharmaceutique en insistant sur la production locale de savoirs.

\section{RÉFÉRENCES BIBLIOGRAPHIQUES}

Alter J.S., 2005, Asian medicine and globalization, Philadelphia, University of Pennsylvania Press.

Banerjee M., 2004, Local knowledge for world market. Globalising ayurveda, Economic and Political Weekly, XXXIX, 3-9, 89-93.

Banerjee M., 2008, Ayurveda in modern India: standardization and pharmaceuticalization, In : Wujastik D., Smith F., eds, Modern and global Ayurveda. Pluralism and paradigms, Albany, State University of New York Press, 201 214.

Bode M., 2008, Taking traditional knowledge to the market. The modern image of the Ayurvedic and Unani industry 1980-2000, Hyderabad, Orient Longman.

Gofraind T., Ardaillou R.M., 2007, Approche classique et innovation pour la recherche de nouveaux médicaments, Bulletin de l'Académie Nationale de Médecine, 191, 4-5, 715-726.

Hsu E., 2001, Innovation in Chinese medicine, Cambridge, Cambridge University Press.

Hsu E., 2009, Chinese propriety medicines: An "alternative modernity?" The case of the anti-malarial substance artemisinin in East Africa, Medical Anthropology, 28, 2, 111-140.

Janes C.R., 2002, Buddhism, science, and market: The globalisation of Tibetan medicine, Anthropology and Medicine, 9, 3, 267-289.

Knauft B., 2002, Critically modern: An introduction, In : Knauft B., ed., Critically modern: alternatives, alterities, anthropologies, Bloomington, Indiana University Press, 1-53.

Lau K., 2000, New age capitalism: making money East of Eden, Philadelphia, University of Pennsylvania Press.

Lei S.H., 1999, From changshan to a new anti-malarial drug. Re-networking Chinese drugs and excluding Chinese doctors, Social Studies of Science, 29, 3, 323-358.

Leslie C., 1976, Asian medical systems: a comparative study, Berkeley, University of California Press. 
Pordié L., 2008, Tibetan medicine today: neo-traditionalism as an analytical lens and a political tool, In : Pordié L., ed., Tibetan medicine in the contemporary World. Global politics of medical knowledge and practice, London and New York, Routledge, 3-32.

Pordié L., 2011a, Savoirs thérapeutiques asiatiques et globalisation, Revue d'Anthropologie des Connaissances, 5, 1, 1-12.

Pordié L., 2011b, Se démarquer dans l'industrie du bien-être. Transnationalisme, innovation et indianité, Anthropologie et Santé, 3 ; http://anthropologiesante.revues.org/805

Pordié, L., Gaudillière, J.P., 2012, Les voies indiennes de l'innovation pharmaceutique. A propos des médicaments ayurvédiques de dernière génération, Autrepart, 64, sous presse.

Scheid V., 2007, Currents of tradition in Chinese medicine (1626-2006), Seattle, Eastland Press.

Selby M.A, 2005, Sanskrit gynecologies in postmodernity: the commoditization of Indian medicine in alternative medical and New Age discourses on women's health, In : Alter J., ed., Asian medicine and globalization, Philadelphia, University of Pennsylvania Press, 120-131.

Spitzer D., 2009, Ayurvedic tourism in Kerala, Local identities and global market, In : Winter T., Teo P., Chang P.C., eds, Asia on tour. Exploring the rise of Asian tourism, London and New York, Routledge, 138-149.

Zimmermann F., 2011, Du phlegmon à l'azadirachtine. Représentations indiennes des maladies et bioprospection, In : Godelier M., ed., Maladie et santé selon les sociétés et les cultures, Paris, PUF, 53-74.

Zysk K., 2001, New Age Ayurveda or what happens to Indian medicine when it comes to America, Traditional South Asian Medicine, 6, 10-26. 